\title{
Introduction
}

\section{Carlo Carraro}

The formation of coalitions is a widespread phenomenon in old and contemporary economies. A coalition emerges when a group of citizens form a club, when firms set up a cartel, when countries agree on free trade. A firm can be seen as a coalition, the outcome of international negotiations on environmental matters or on other economic issues are coalitions (for example, a monetary union is a coalition), a family can also be classed as a coalition. Research joint ventures and federal states are also coalitions. Relevant economic coalitions are numerous and cover the most important aspects of economic life.

Economists and game-theorists have been working on coalition theory for many years. Most recently, their main goal was not to understand how a group of economic agents share the benefit of forming a coalition (as in Nash's seminal work and subsequently in Rubinstein's important characterization). On the contrary, the main goal was to understand whether economic agents have an incentive to form a coalition, that is, whether they actually decide to form a coalition. Therefore, the issue is not whether the coalition is profitable and how this profit can be shared among coalition members, but whether the coalition is stable or self-enforcing, that is, which economic agents join the coalition and why. ${ }^{1}$ Most importantly, the reformulation of the problem of coalition formation in non-cooperative terms can handle the issue of competition between coalitions which in traditional cooperative theory was largely ignored (see the introduction to Kovalenkov and Wooders in this volume).

The non-cooperative approach to coalition formation can be loosely described as follows (see the chapters by Bloch and Yi in this book for a detailed presentation). The decision of whether or not to join the coalition is a 'meta-decision' that precedes the cooperative strategy characterising the behaviour of coalition members once the coalition is formed. This metadecision is taken non-cooperatively. Thus, the outcome of the meta-game in which agents decide whether or not to join a coalition - and which coalition - is identified via a non-cooperative equilibrium concept. In addition, the partition function approach (surveyed by $\mathrm{Yi}$ in this volume) can be applied and interactions between coalitions are taken into account. The 
main objective of this book is to provide an overview of these recent advances in the non-cooperative game-theoretic approach to coalition formation.

In recent years, there have indeed been several attempts to provide a comprehensive theory of coalition formation using a non-cooperative approach. Many equilibrium concepts have been proposed and their implications on the coalition structure have been derived. The emergence of coalition structures with multiple coalitions has been stressed /highlighted. The size of these coalitions and their sensitivity to changes in the economic environment have been analysed. Applications of these new theories to industrial organizations (extending traditional cartel theory and RJVs theory), environmental economics (mainly focusing on international environmental treaties), trade theory (free trade areas, custom unions), public finance (public and club good provisions) and so on have been proposed.

This book is the first attempt to provide an overview of the main theoretical results and of their applications. Chapters 2-6 survey the different approaches to endogenous (that is, non-cooperative) coalition formation, discuss and compare the diversity of equilibrium concepts, and analyse the main features of the emerging coalition structures. Each chapter discusses different applications of the theory proposed and how these applications improve our understanding of relevant economic problems.

Most applications discussed in this book deal with global and local public goods and with club goods. The focus on public and club goods is related to the well-known difficulty of achieving efficient outcomes in the presence of free-riding incentives. The chapters of this book address this difficulty by using the non-cooperative approach to coalition formation where a coalition can be seen as a group of agents who cooperate to provide a club or public good - and show that in general cooperation emerges at the equilibrium as a result of independent and voluntary decisions taken by each agent.

Before presenting the non-cooperative approach to coalition formation and its applications, this book highlights some important advances achieved by using the cooperative approach to coalition formation. Therefore, the first chapter of this book, written by Alexander Kovalenkov and Myrna Holtz Wooders, is a bridge between the cooperative and noncooperative approach. Its starting point is the relationship between the core and competitive economies, two concepts apparently antithetic. The authors note that in the analysis of models of private goods exchange economies, price-taking competitive equilibrium outcomes and cooperative outcomes - particularly the core - have played a central role. However, in cases like network economies, clubs, coalition production, matching models, and local public goods, the analogue of price-taking equilibrium is 
not clear. Moreover, price-taking equilibrium depends on the details of the economic model. This and the close relationship between the core and price-taking equilibrium in a variety of contexts and their equivalence in large economies, motivates the study of the core as an analogue of the competitive equilibrium, especially in the context of games with many players. The concept of the core encounters difficulty, however, because apart from idealised situations it may well be empty. This has given rise to a literature demonstrating that cooperative games with many players have nonempty approximate cores and the approximation can become very close as the games become large. Moreover, there are outcomes in approximate cores that treat most or all similar players nearly identically, a property of pricetaking equilibrium outcomes.

The chapter by Kovalenkov and Wooders explores all the aforementioned outcomes and results and also provides interesting examples and applications. In particular, Kovalenkov and Wooders treat games with side payments and present some recent results for this specific case. Their analysis can be used, for example, to deal with economies with local public goods - public goods subject to exclusion and congestion. Their results can also be applied to the case of multijurisdictional economies, where it is natural to allow an individual to belong to several jurisdictions and where jurisdictions may overlap. For example, an individual could be a member of a marriage, an employee of a University, a citizen of a country and a member of a large international environmental organization. Through application of their results, Kovalenkov and Wooders demonstrate that, when individuals belong to multiple clubs and when small groups are effective, then large club economies have nonempty approximate cores.

As said, the theoretical framework adopted by Kovalenkov and Wooders is still a cooperative one. Therefore, the main problem with Kovalenkov and Wooders' framework is that it allows the payoffs to a coalition to be affected by the actions of the coalition, but the choices of a coalition are assumed to have no effect on the payoffs of the complementary coalitions. Suppose, for example, coalitions form to produce pure public goods so that the goods produced by one coalition can be consumed by the entire player set. Each coalition is then affected by the actions chosen by members of the complementary coalitions. This situation cannot be dealt with using Kovalenkov and Wooders' results. Therefore, the cooperative game framework is most applicable to situations where what a coalition can achieve is independent of the actions of the complementary coalitions.

Extensions of the theory of coalition formation to situations in which the opportunities available to a group of players depend on the activities of the non-members of the group have been recently proposed in several papers (see, for example, Ray and Vohra, 1997, 1999; Yi, 1997; Bloch, 
1996). The remaining chapters of this book present the main results achieved within the non-cooperative approach to coalition formation.

Chapter 2, Non-cooperative models of coalition formation in games with spillovers by Francis Bloch, is a comprehensive survey of the noncooperative approach to coalition formation. In the first part of his survey, Bloch analyses in particular the role of spillovers between coalitions and the different representations of games with externalities. He emphasizes the limits of the classical cooperative approach based on the characteristic function and shows how various partition function games can be derived. As said, this approach has the advantage of capturing the possibility of externalities across coalitions, because it allows the change in the coalition structure to affect the values of all coalitions, whereas the characteristic function approach cannot incorporate such externalities across coalitions.

Bloch then discusses a first class of non-cooperative games where all agents simultaneously announce their decision to cooperate. The various outcome rules for the simultaneous announcement game, and the related different solution concepts, are analysed and discussed. In the second part of his chapter, Bloch analyses a second class of games where agents announce sequentially their decision to participate in the coalition. He compares different specifications of the extensive form games reflect differences in the procedures of coalition formation.

Bloch also studies some cooperative solution concepts that were proposed to analyse the stability of coalition structures in games with spillovers. While those stability concepts are not based on non-cooperative processes, they provide interesting benchmarks and give new insights to the study of coalition formation with spillovers.

The third part of the survey is devoted to economic applications. Bloch distinguishes between games with positive and negative spillovers. Games with positive spillovers are games where the formation of a coalition increases the payoff of external members. The formation or cartels and the provision of public goods are examples of games with positive spillovers. Games with negative spillovers are games where the formation of a coalition is harmful to external members. For example, the formation of trade unions or associations of firms are games with negative spillovers. The final part of the survey discusses the most recent contributions to the study of coalition formation with spillovers, and outlines some open questions to be addressed in future research.

Chapter 3, Endogenous formation of economic coalitions: a survey of the partition function approach by Sang-Seung Yi, also emphasizes how coalition formation among autonomous agents is an integral part of economic activity. Examples are taken from industrial organization, international trade and environmental economics. In industrial organizations, 
Japanese firms have established R\&D alliances such as the Very Large Scale Integration (VLSI) consortium and the Fifth Generation Computer Project with the purpose of gaining an edge on international competition. In international trade, there has been a resurgence of regionalism. Casual observation suggests that the world is moving towards the formation of three major trading blocs consisting of the European Union (EU), the North American Free Trade Agreements (NAFTA) zone, and a potential Asian bloc. In environmental economics, policy coordination on greenhouse gas emissions through the 1995 Rio Convention and the 1997 Kyoto Protocol has heightened both economists' and policy makers' interest in the stability of global environmental agreements. Economic coalitions such as research joint ventures and free-trade areas have two distinguishing characteristics. First, several coalitions coexist and compete with each other. Second, the formation, expansion and merger of economic coalitions typically create externalities on non-members.

Yi surveys recent developments in the non-cooperative theory of coalition formation that emphasize these two characteristics of economic alliances: the formation of multiple, competing coalitions that create externalities on nonmembers. His work is also based on the partition function approach to coalition formation. This approach is presented at the beginning of his survey. Yi then reviews several rules of coalition formation that have been proposed in the literature and discusses the equilibrium outcomes of specific coalition games.

In the fourth chapter, Farsightedness in coalition formation by Marco Mariotti and Licun Xue, an extension of the partition function approach is proposed. In particular, new equilibrium concepts, based on the assumed enhanced farsightedness of coalition members, are discussed. Loosely speaking, a coalition is farsighted when it looks ahead at the ultimate consequences of its actions. For example, if a sub-coalition breaks up from a larger coalition, will this cause further break-ups? And how will the members who are excluded from the deviating coalition react? To deal with these questions, Mariotti and Xue start from von Neumann and Morgenstern's stable set for cooperative games and from Greenberg's (1990) generalization of this stability notion. ${ }^{2}$ One of the situations studied by Greenberg is the 'coalitional contingent threat situation' associated with a strategic game, a negotiation process in which coalitions make open threats contingent on the strategy profile under consideration. This situation is subsequently studied in Chwe (1994) and others (for example, Mariotti, 1997; Xue, 1998, 2000). The results obtained by these authors are surveyed in this chapter. In addition, this chapter analyses several applications of the above literature, including bargaining theory, repeated games, theory of unemployment and environmental negotiations. 
Chapter 5 focuses on the stability of coalitions that have already formed. This chapter, entitled Stable coalitions and written by Carlo Carraro and Carmen Marchiori, examines recent theoretical developments of the theory of coalition stability. It focuses on the relationship between the incentives to defect from a coalition, the size of the resulting equilibrium coalition structure, and the different assumptions on membership rules, coalition behaviour, players' conjectures and so on. The paper considers several cases: simultaneous vs. sequential moves, linear vs. circular order of moves, Nash vs. rational conjectures, open vs. exclusive membership, monotonic vs. non monotonic payoff functions, and orthogonal vs. nonorthogonal reaction functions. The profitable and stable coalitions are derived for each possible configuration of the rules of the game, the payoff functions and the membership rules. The results show that the size of the profitable and stable coalition highly depends on the chosen configuration and that the equilibrium outcome ranges from a small coalition with a few signatories to full cooperation. This chapter explores under which conditions a large stable coalition is likely to emerge, and identifies the institutional setting that favours the emergence of such coalition.

The final chapter, Endogenous coalition formation in global pollution control: a partition function approach by Michael Finus and Bianca Rundshagen, expands on the work by Carraro and Marchiori by allowing for multiple coalitions. Again equilibrium coalitions are derived under different 'rules of the game' and compared with each other. Finus and Rundshagen consider internal and external stability and equilibrium binding agreements in the cartel formation game, coalition-proof and strong Nash equilibria in the open-membership game and in the exclusive membership $\alpha$ - and $\gamma$-games, subgame-perfect equilibria in the sequential move unanimity game and in the equilibrium binding agreement game. They show that farsightedness and exclusive membership leads to a more concentrated coalition structure, implying lower global emissions and higher global welfare. Moreover, they evaluate the different coalition formation games with respect to their ability to explain existing international environmental agreements and with respect to their theoretical consistency.

The six chapters of this book provide a substantial overview of recent advances in coalition theory. They are written by some of the most important authors in the field and enable the reader to compare different approaches and results. In addition, they show the many applications of coalition theory and its ability to address difficult economic problems (for example, the provision of global public goods). Therefore, this book provides a new set of tools that can be applied to several economic issues (a phenomenon which is already taking place and which confirms the timing of this book). 


\section{NOTES}

1. Of course, the study of this latter issue also depends on the way in which the coalition benefit is shared among coalition members, but the two are different and are analysed with different economic tools.

2. Greenberg's contribution spawned literature which aimed to extend, modify and apply the original theory. For example, Chwe (1994) considers a social environment which, although less general than a situation, is sufficiently flexible to integrate the presentation of some negotiation processes associated with cooperative, strategic and extensive games. Chwe's main objective is to offer a notion that captures foresight and is likely to be nonempty. Xue (1998) shows that Chwe's notion captures only partial foresight and offers a solution concept that captures perfect foresight using Greenberg's framework.

\section{REFERENCES}

Bloch, F. (1996), 'Sequential formation of coalition in games with externalities and fixed payoff division', Games and Economic Behaviour, 14, 90-123.

Chwe, M.S.Y. (1994), 'Farsighted coalition stability', Journal of Economic Theory, 54, 299-325.

Greenberg, J. (1990), The Theory of Social Situations, Cambridge: Cambridge University Press.

Mariotti, M. (1997), 'A model of agreements in strategic form games', Journal of Economic Theory, 73, 196-217.

Ray, D. and R. Vohra (1997), 'Equilibrium binding agreements', Journal of Economic Theory, 73, 30-78.

Ray, D. and R. Vohra (1999), 'A theory of endogenous coalition structure', Games and Economic Behaviour, 26, 286-336.

Xue, L. (1998), 'Coalitional stability under perfect foresight', Economic Theory, 1, 603-27.

Xue, L. (2000), 'Negotiation proof nash equilibrium', International Journal of Game Theory, 29, 339-57.

Yi, S. (1997), 'Stable coalition structures with externalities', Games and Economic Behaviour, 20, 201-23. 
Carlo Carraro - 9781781009888 Downloaded from PubFactory at 04/26/2023 01:14:59PM via free access 